\title{
Probable detection of an eruptive filament from a superflare on a solar-type star
}

\author{
Kosuke Namekata ${ }^{1,2,3 \varpi}$, Hiroyuki Maehara4 , Satoshi Honda ${ }^{5}$, Yuta Notsu ${ }^{6,7,8}$, Soshi Okamoto', \\ Jun Takahashi ${ }^{5}$, Masaki Takayama5, Tomohito Ohshima5, Tomoki Saito5, Noriyuki Katoh ${ }^{5,9}$, \\ Miyako Tozuka5, Katsuhiro L. Murata ${ }^{10}$, Futa Ogawa ${ }^{10}$, Masafumi Niwano ${ }^{10}$, Ryo Adachi ${ }^{10}$, \\ Motoki Oeda ${ }^{10}$, Kazuki Shiraishi ${ }^{10}$, Keisuke Isogai ${ }^{2,11}$, Daikichi Seki 1,2,12, Takako T. Ishii ${ }^{2}$, Kiyoshi Ichimoto ${ }^{2}$, \\ Daisaku Nogami' and Kazunari Shibata ${ }^{13,14}$
}

\begin{abstract}
Solar flares are often accompanied by filament/prominence eruptions $\left(-10^{4} \mathrm{~K}\right.$ and $\left.\sim 10^{10-11} \mathrm{~cm}^{-3}\right)$, sometimes leading to coronal mass ejections that directly affect the Earth's environment ${ }^{1,2}$. 'Superflares' are found on some active solar-type (G-type main-sequence) stars $^{3-5}$, but the filament eruptioncoronal mass ejection association has not been established. Here we show that our optical spectroscopic observation of the young solar-type star EK Draconis reveals evidence for a stellar filament eruption associated with a superflare. This superflare emitted a radiated energy of $2.0 \times 10^{33} \mathrm{erg}$, and a blueshifted hydrogen absorption component with a high velocity of $-510 \mathrm{~km} \mathrm{~s}^{-1}$ was observed shortly afterwards. The temporal changes in the spectra strongly resemble those of solar filament eruptions. Comparing this eruption with solar filament eruptions in terms of the length scale and velocity strongly suggests that a stellar coronal mass ejection occurred. The erupted filament mass of $1.1 \times 10^{18} \mathrm{~g}$ is ten times larger than those of the largest solar coronal mass ejections. The massive filament eruption and an associated coronal mass ejection provide the opportunity to evaluate how they affect the environment of young exoplanets/the young Earth ${ }^{6}$ and stellar mass/angular momentum evolution?.
\end{abstract}

Solar flares, filament eruptions and coronal mass ejections (CMEs) are thought to be caused by a common magnetohydrodynamic process, though not all of them are necessarily observed in the same event. Magnetic reconnection is a key energy release mechanism for flares, which are thought to be sometimes triggered by the instability of cool filaments in active regions ${ }^{1}$. Recently, it has been discussed that much larger 'superflares' that release the energy of more than $10^{33} \mathrm{erg}$ (ten times the largest solar flares, $\sim 10^{32} \mathrm{erg}$ ) can occur-or have occurred relatively recently-even on the $\operatorname{Sun}^{3-5,8}$. Superflares may produce much larger CMEs than the largest solar flares, which can severely affect the environment, habitability and development of life around young and intermediate-age stars ${ }^{6}$. However, superflares on solar-type stars have been mainly detected by optical photometry (for example, the Kepler space telescope) ${ }^{3}$.
Therefore, no observational indication of filament eruptions/CMEs has been reported for solar-type stars. Optical spectroscopic observations are a promising way to detect stellar filament eruptions, which can be indirect evidence of CMEs. However, for solar-type stars, optical spectra of superflares have never been obtained.

EK Draconis (EK Dra) is known to be an active young solar-type star (a G-type, zero-age main-sequence star with an effective temperature of 5,560-5,700 K and age of 50-125 Myr; ref. ${ }^{9}$ ) that exhibits frequent UV stellar flares ${ }^{10,11}$ and gigantic starspots at low-high latitudes ${ }^{9}$. We conducted optical spectroscopic monitoring of EK Dra for 19 nights between 21 January 2020 and 15 April 2020, simultaneously with optical photometry from the Transiting Exoplanet Survey Satellite (TESS) ${ }^{12}$. Time-resolved neutral-hydrogen $\mathrm{H} \alpha$-line spectra at $6,562.8 \AA$ (radiation from cool plasma of a few times $10,000 \mathrm{~K}$ ) were spectroscopically observed at the $3.8 \mathrm{~m}$ Seimei telescope ${ }^{13}$ and the $2 \mathrm{~m}$ Nayuta telescope. In this campaign, we succeeded in obtaining optical spectra of large superflares on a solar-type star. The superflare that occurred on 5 April 2020 was simultaneously observed using TESS photometry in white light $(\sim 6,000-10,000 \AA)$ and ground-based spectroscopy in the $\mathrm{H} \alpha$ line (Fig. 1a,b and Extended Data Fig. 1). The H $\alpha$ brightening was associated with the TESS white-light flare, which lasted $16 \pm 2 \mathrm{~min}$. The radiated bolometric energy of the TESS white-light flare is estimated to be $2.0 \pm 0.1 \times 10^{33} \mathrm{erg}$ (20 times the most energetic solar flares), and the radiated $\mathrm{H} \alpha$-line energy was $1.7 \pm 0.1 \times 10^{31} \mathrm{erg}$; thus, the flare is classified as a superflare.

After the impulsive phase, the TESS white-light intensity returned to its pre-flare level. However, the equivalent width (hereafter $\mathrm{EW}$ ) of $\mathrm{H} \alpha$ (the wavelength-integrated $\mathrm{H} \alpha$ emission normalized by the continuum level) became lower than the pre-flare level (that is, it displayed enhanced absorption), returning to the pre-flare level after approximately $2 \mathrm{~h}$ (Fig. 1b). The blueshifted $\mathrm{H} \alpha$ absorption component with a maximum central velocity of about $-510 \mathrm{~km} \mathrm{~s}^{-1}$ and a half-width of $\pm 220 \mathrm{~km} \mathrm{~s}^{-1}$ appeared soon after the superflare. The velocity gradually slowed with time, and a redshifted absorption component appeared at a few times $10 \mathrm{~km} \mathrm{~s}^{-1}$

'Department of Astronomy, Kyoto University, Sakyo, Kyoto, Japan. ${ }^{2}$ Astronomical Observatory, Kyoto University, Sakyo, Kyoto, Japan. ${ }^{3}$ ALMA Project, National Astronomical Observatory of Japan, NINS, Mitaka, Tokyo, Japan. ${ }^{4}$ Okayama Branch Office, Subaru Telescope, National Astronomical Observatory of Japan, NINS, Asakuchi, Okayama, Japan. ${ }^{5}$ Nishi-Harima Astronomical Observatory, Center for Astronomy, University of Hyogo, Sayo, Hyogo, Japan. ${ }^{6}$ Laboratory for Atmospheric and Space Physics, University of Colorado Boulder, Boulder, CO, USA. ${ }^{7}$ National Solar Observatory, Boulder, CO, USA. ${ }^{8}$ Department of Earth and Planetary Sciences, Tokyo Institute of Technology, Meguro, Tokyo, Japan. ${ }^{9}$ Graduate School of Human Development and Environment, Kobe University, Nada, Kobe, Japan. ${ }^{10}$ Department of Physics, Tokyo Institute of Technology, Meguro, Tokyo, Japan. "Department of Multi-Disciplinary Sciences, Graduate School of Arts and Sciences, The University of Tokyo, Meguro, Tokyo, Japan. ${ }^{12}$ Graduate School of Advanced Integrated Studies in Human Survivability, Kyoto University, Sakyo, Kyoto, Japan. ${ }^{13}$ Kwasan Observatory, Kyoto University, Yamashina, Kyoto, Japan.

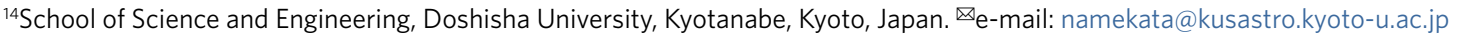



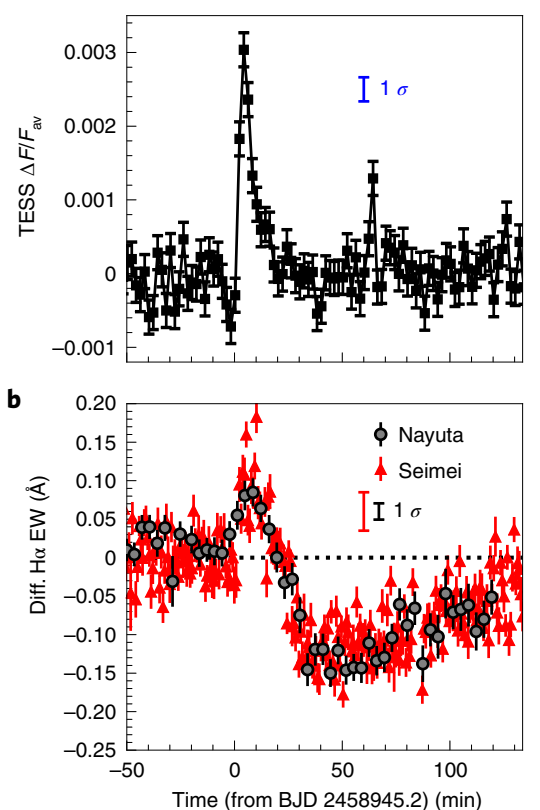



d

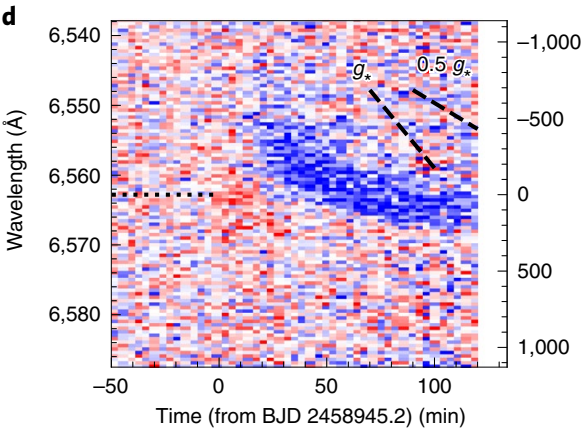



Fig. 1 light curves and spectra of a superflare on EK Dra. a, The light curve observed by TESS in white light ( 6,000-10,000 $\AA$ ) on BJD (barycentric Julian day) 2458945.2 (5 April 2020). The individual points represent the stellar flux normalized by the averaged value with the pre-flare level subtracted. The $1 \sigma$ value of the pre-flare light curve ( $-150 \mathrm{~min}$ to $0 \mathrm{~min}$ ) is shown in blue. $\mathbf{b}$, Light curves of the $\mathrm{H} \alpha \mathrm{EW}$ observed using the medium-dispersion spectrograph MALLS (Medium and Low-Dispersion Long-Slit Spectrograph) at the Nayuta telescope (grey circles) and the low-dispersion spectrograph KOOLS-IFU (Kyoto Okayama Optical Low-Dispersion Spectrograph with optical-fibre Integral Field Unit) installed at the Seimei telescope (red triangles) during the same observing period as in $\mathbf{a}$. The $\mathrm{H} \alpha$ emissions were integrated within $\pm 10 \AA$ from the $\mathrm{H} \alpha$ line centre $(6,562.8 \AA)$ after dividing by the continuum level, and the pre-flare level was subtracted. The positive and negative values represent emission and absorption, respectively, compared with the pre-flare level. The $1 \sigma$ value of the pre-flare light curve $(-150 \mathrm{~min}$ to $0 \mathrm{~min})$ is plotted in red and black for Seimei and Nayuta data, respectively. c,d, Two-dimensional H $\alpha$ spectra obtained using the Seimei telescope (c) and the Nayuta telescope (d). The red and blue colours correspond to emission and absorption, respectively. The dashed lines indicate the stellar surface gravity $\left(g_{*}\right)$ and half of the surface gravity $\left(0.5 g_{*}\right)$. c and $\mathbf{d}$ share the upper colour bar. e, Temporal evolution of the pre-flare-subtracted $\mathrm{H} \alpha$ spectra observed using the Seimei telescope (red) and the Nayuta telescope (black), with the spectra shifted by constant values for clarity. The spectra are binned in time, and the integration periods correspond to the horizontal axes of a-d. The intensities are normalized by the stellar continuum level. The vertical dotted line indicates the $\mathrm{H} \alpha$ line centre, and the horizontal dotted lines indicate the zero levels for each spectrum. The $1 \sigma$ error bar around the line core, based on the residual scattering in the line wing, is also shown.

(Fig. 1c-e and Extended Data Figs. 2a and 3a). Both ground-based spectroscopic observations simultaneously recorded the same spectral change, demonstrating that low-temperature and high-density neutral plasma above the stellar disk moves at high speed toward the observer before some parts finally start to fall back to the surface. In addition, the deceleration is not monotonic: it was $0.34 \pm 0.04 \mathrm{~km} \mathrm{~s}^{-2}$ in the initial phase, dropping to $0.016 \pm 0.008 \mathrm{~km} \mathrm{~s}^{-2}$ in the later phase (Fig. 1c,d and Extended Data Fig. 3b). This is interpreted in terms of changes in the height of the ejected mass. The observed deceleration is in good agreement with that due to the surface gravity of approximately $0.30 \pm 0.05 \mathrm{~km} \mathrm{~s}^{-2}$ ( ref. $^{9}$ ), although the initial value is slightly larger.

How much do the stellar spectral changes obtained here actually resemble those of solar filament eruptions? Blueshifted $\mathrm{H} \alpha$ absorption profiles are often observed from solar filament eruptions ${ }^{1,14}$. As in Fig. 2, we generated spatially integrated Ho spectra of a solar flare/filament eruption that occurred on the solar disk using the SMART (Solar Magnetic Activity Research Telescope) data ${ }^{15}$ (Extended Data Fig. 4 and Supplementary Video 1). We converted to the full-disk pre-flare-subtracted spectra by multiplying by the partial-region/full-disk ratio (that is, virtual Sun-as-a-star spectra). We found that the blueshifted absorption component at approximately $100 \mathrm{~km} \mathrm{~s}^{-1}$ was predominant soon after the solar flare, and the spatially integrated $\mathrm{H} \alpha \mathrm{EW}$ showed enhanced absorption
(Fig. 2a). These blueshifted profiles are unequivocally due to the filament eruption. Later, the blueshifted component decelerated and gradually turned into slow, redshifted absorption (Fig. 2b,c). The H $\alpha$ EW returned to the pre-flare level in approximately $40 \mathrm{~min}$ (Fig. 2a). Although the energy scales and velocities are different, the solar data strongly resemble the spectral changes in the superflare on EK Dra (see Supplementary Information for another event). This similarity suggests that the stellar phenomenon is the same as the simply magnified picture of the solar filament eruption.

A filament eruption is the only explanation for the blueshifted absorption component on EK Dra by solar analogy ${ }^{1}$. The hypothesis that the blueshifted absorption on EK Dra might come from up-/downflow in flare kernels must be rejected because they never show $\mathrm{H} \alpha$ absorption ${ }^{16,17}$. Also, downflow in cooled magnetic loops (known as post-flare loops ${ }^{14}$ shows redshifted absorption, so they cannot explain the blueshifted absorption. (However, the redshifted absorption in EK Dra in the later phase might be caused by post-flare loops $^{14}$.) Rotational visibilities of prominences or spots also are not adequate to explain it, since the rotation speed of EK Dra is only $16.4 \pm 0.1 \mathrm{~km} \mathrm{~s}^{-1}\left(\right.$ ref. $\left.^{9}\right)$. Thus, we concluded that we detected a stellar filament eruption on the solar-type star.

Some observational signatures for stellar filament eruptions or CMEs have been reported previously for cooler K-M dwarfs ${ }^{18-22}$ and evolved giant stars $^{23}$ (see Methods and refs. ${ }^{6,24}$ for reviews). 
a

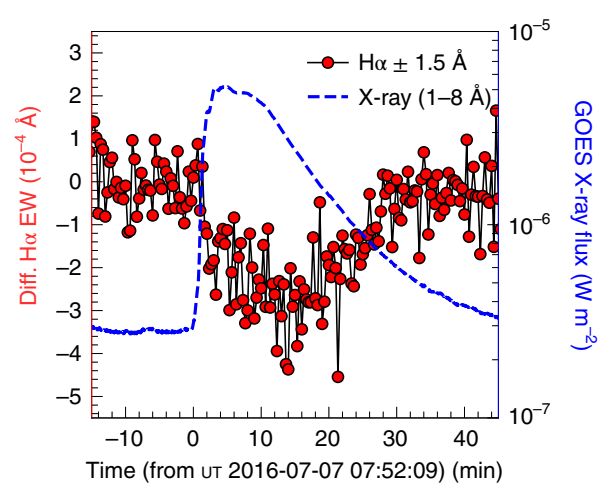

b

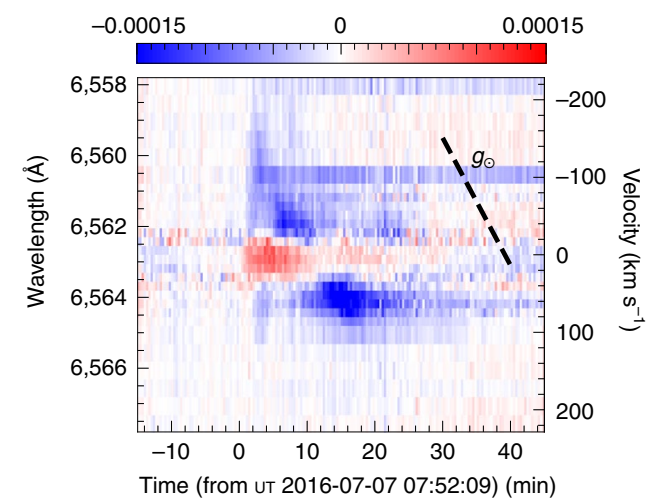

c

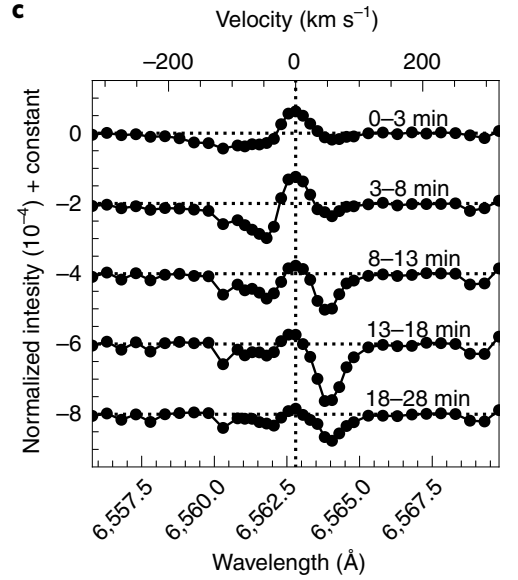

Fig. 2 | The space-integrated light curves and spectra of a C5.1-class solar flare and filament eruption on 7 July 2016, observed with the SDDI (Solar Dynamics Doppler Imager) installed at SMART. a, GOES (Geostationary Operational Environmental Satellite) soft X-ray (1-8 ) and H $\alpha$ EW light


centre $(6,562.8 \AA)$ and were divided by the full-disk continuum level, and the pre-flare level was subtracted. Time 0 is the time when the flare begins. b. Two-dimensional pre-flare-subtracted $\mathrm{H} \alpha$ spectra. The red and blue colours correspond to emission and absorption compared with the pre-flare levels, respectively. The dashed line indicates gravity at the solar surface. c, Temporal evolution of the pre-flare-subtracted $\mathrm{H} \alpha$ spectrum shifted vertically by constant values for clarity. The $\mathrm{H} \alpha$ spectra were produced by integrating the data over a large enough region to cover the flaring area (Extended Data Fig. 4). The intensities are normalized by the total solar continuum level. The vertical dotted line indicates the H $\alpha$ line centre, and the horizontal dotted lines indicate the zero levels for each spectrum.

The observation of a giant star shows a blueshifted X-ray emission line of $90 \mathrm{~km} \mathrm{~s}^{-1}$ in the post-flare phase and hotter CME is proposed as a possible explanation ${ }^{23}$. Recently, X-ray/extreme UV dimmings have been reported as indirect evidence of stellar CMEs on $\mathrm{K}-\mathrm{M}$ dwarfs ${ }^{22}$. In M-dwarf flares, many blueshifted Balmer/UV line emission components have been reported ${ }^{18-21,24}$, which are interpreted as filament eruptions. Some M-dwarf flares share properties with the eruption on EK Dra: the blueshift emissions have high velocities of hundreds of kilometres per second, and some exhibit velocity changes and appear after the impulsive phase ${ }^{20,21}$. For M-dwarf events, the number of studies reporting highly time-resolved velocity variations of blueshift components is still insignificant ( $\sim 5$ min cadence), and a simultaneous white-light flare has never been detected. Our detection of a stellar filament eruption is reliable because we provided solar counterparts, highly time-resolved spectra ( $~ 50$ s cadence) and a simultaneous TESS white-light flare.

What properties does the filament eruption on EK Dra have? The maximum observed velocity of the blueshifted component was $\sim-510 \mathrm{~km} \mathrm{~s}^{-1}$ with a width of $220 \mathrm{~km} \mathrm{~s}^{-1}$. This is larger than the typical velocities of solar filament eruptions (10-400 $\mathrm{km} \mathrm{s}^{-1}$ ) associated with $\mathrm{CMEs}^{2}$, although it is a little smaller than the escape velocity at the surface on EK Dra $\left(\sim 670 \mathrm{~km} \mathrm{~s}^{-1}\right)$. The cool plasma reached at least $\sim 1.0$ stellar radius from the stellar surface (or the initial height) as derived by integrating the velocity over time (or $\sim 3.2$ stellar radii from the stellar surface on the basis of the deceleration rates). In this case, a projection angle of at most $45^{\circ}$ can be allowed when we assume that the event occurs on the disk centre. On this projection angle, the velocity can be up to $\sim-720 \mathrm{~km} \mathrm{~s}^{-1}$, so there is a possibility that the velocities of some components of the EK Dra eruption could exceed the escape velocity. However, it should be noted that there are weak redshifted components with a velocity of a few $10 \mathrm{~km} \mathrm{~s}^{-1}$ in the late phase, indicating that some materials fell back to the star. This is often observed in the case of solar filament eruptions with $\mathrm{CMEs}^{25}$.

The filament area is estimated to be $1.6 \times 10^{21} \mathrm{~cm}^{2}(5.6 \%$ of the stellar disk), and the erupted mass is calculated to be $1.1_{-0.9}^{+4.2} \times 10^{18} \mathrm{~g}$ on the basis of the absorption components. The mass is more than ten times larger than those of the largest solar $\mathrm{CMEs}^{26,27}$ (it should be noted that the mass can be somewhat under-/overestimated; Methods). This mass estimate is in reasonable agreement with those predicted from empirical $^{26,27}$ and theoretical ${ }^{28}$ solar scaling relations between CME mass and flare energy within the error bars $\left(\sim 9.4_{-2.4}^{+3.2} \times 10^{16}\right.$ and $3.1_{-1.1}^{+1.6} \times 10^{17}$ g for refs. ${ }^{27}$ and ${ }^{26}$, respectively) (Fig. 3a). This suggests that the stellar filament eruption can share a common underlying mechanism with smaller-scale filament eruptions/CMEs (that is, magnetic energy release ${ }^{1,28}$ ) although the absolute values of most physical quantities are very different.

Moreover, the kinetic energy is calculated to be $3.5_{-3.0}^{+14.0} \times 10^{32} \mathrm{erg}$, which is $16 \%$ of the radiation energy in white light. The magnetic energy stored around the starspots on EK Dra can be at least $8.0 \times 10^{35} \mathrm{erg}$, which is enough to produce superflares and filament eruptions with energy of $\sim 10^{33} \mathrm{erg}$. In addition, this value is slightly smaller than those extrapolated from the solar CME scaling law $\left(4.8_{-0.9}^{+1.1} \times 10^{33} \mathrm{erg}\right.$; ref. $\left.{ }^{27}\right)$ (Fig. $3 \mathrm{~b}$ ), which is similar to the filament eruption/CME candidates on other $\operatorname{stars}^{24}$. In previous studies, it has been argued that kinetic energy can be reduced by overlying magnetic fields ${ }^{24,29}$. The deceleration of our events was a few times $10 \%$ larger than the stellar gravity (Extended Data Fig. 3b). The strong magnetic fields on EK Dra have been reported before ${ }^{9}$ and may support the above explanations. However, its small kinetic energy can also be understood through a solar analogy: the velocities of (lower-lying) filament eruptions are usually four to eight times lower than those of the corresponding (higher-lying) $\mathrm{CMEs}^{2}$, and therefore the kinetic energies of filament eruptions are typically smaller (green symbols in Fig. 3b).

Did a CME occur in this event? Obviously, the line-of-sight velocity of $\sim 510 \mathrm{~km} \mathrm{~s}^{-1}$ was lower than the escape velocity and some masses fell back, which may indicate a so-called 'failed' filament eruption ${ }^{29}$. However, this does not necessarily mean that a CME did not occur, again by solar analogy. In fact, the erupted filaments often fall back to the Sun when CMEs happen. For example, a well studied solar event on 7 June 2011 involved a $200-600 \mathrm{~km} \mathrm{~s}^{-1}$ filament eruption where much filamentary material fell back to the Sun, but some mass clearly escaped as a CME with velocities of $\sim 1,000 \mathrm{~km} \mathrm{~s}^{-1}$ (ref. ${ }^{25}$ and Supplementary Information). The event 



Fig. 3 | Mass and kinetic energy as a function of flare energy for solar and stellar flares and filament eruptions/CMEs. a, Comparison between bolometric flare energy and ejected mass. The red square represents the superflare on EK Dra, the black crosses denote solar CME data, the green triangles signify data for solar prominence/filament eruptions and surges taken from previous studies and the green plus sign is the solar filament eruption/surges displayed in Fig. 2 and Supplementary Fig. 9 (Velocity, mass, and kinetic energy: solar data), respectively (Table 1). Note that solar 'surges' are jet-like filament eruption phenomena (see Another case of solar flares on 2 April 2017 for an explanation of the surge). The cyan dashed and magenta dotted lines are trend fits for solar CMEs expressed as $M_{C M E s} \propto E^{0.59}$ and $M_{C M E S} \propto E^{0.7}$, respectively (Solar flare energy-CME mass relation and refs. ${ }^{26,27}$ ). b, Comparison between flare bolometric energy and kinetic energy of the erupted mass. The symbols are the same as in $\mathbf{a}$. The cyan dashed line is a fit for solar $C M E s$ expressed as $E_{k i n} \propto E_{x}^{1.05}$. The kinetic energy of eruption on EK Dra is calculated to be $3.5_{-3.0}^{+14.0} \times 10^{32} \mathrm{erg}$, which is outside the error range of the predicted value of $4.8_{-0.9}^{+1.1} \times 10^{33} \mathrm{erg}$ $\left(\right.$ ref. ${ }^{27}$ ). The error bars are derived as the model errors (see 'Velocity, mass and kinetic energy: stellar data').

on EK Dra may correspond to this solar event. In addition, ref. ${ }^{30}$ showed that whether a solar filament eruption leads to a CME can be simply distinguished by a parameter of $\left(V_{r_{\text {- } \max }} / 100 \mathrm{~km} \mathrm{~s}^{-1}\right)$ $(L / 100 \mathrm{Mm})^{0.96}$, where $V_{r_{-} \max }$ is the maximum radial velocity and $L$ is the length scale (Fig. 4 ). When the parameter is more than $\sim 0.8$, the probability that a filament eruption leads to a CME is more than $90 \%$ (ref. ${ }^{30}$ ). The value of the parameter of eruption on EK Dra is $\sim 18$, meaning that our detection of the fast and sizable stellar filament eruption is indirect evidence that mass escapes into interplanetary space as a CME.

Finally, we summarize future directions of our findings (see Supplementary Information for details): It is speculated that the filament eruptions/CMEs associated with superflares can severely affect planetary atmospheres ${ }^{6}$. Our findings can therefore provide a proxy for the possible enormous filament eruptions on young solar-type stars and the Sun, which would enable us to evaluate the effects on the ancient, young Solar System planets and the Earth, respectively. Further, it is also speculated that stellar mass loss due to filament eruptions/CMEs can affect the evolutionary theory of stellar mass, angular momentum and luminosity ${ }^{7,26}$ more importantly than can stellar winds. At present, frequency and statistical properties of CMEs on solar-type stars are unknown, but important insights into these factors will be obtained by increasing the number of samples in the future. 




Fig. 4 | Statistical properties of solar filament eruptions taken from ref. ${ }^{30}$ and comparison with the stellar filament eruption on EK Dra. Comparison between the $V_{r \text { max }}$ and $L$ of filament eruptions. The blue circles and orange crosses indicate the solar filament eruptions with and without CMEs, respectively. The red square corresponds to the stellar filament eruption on EK Dra obtained here (see Stellar velocity, mass and kinetic energy data for the calculation of the length scale). Note that the stellar data are the observed line-of-sight velocities. Since the deceleration of the stellar filament eruption corresponds well to the surface gravity and the absorption component of the filament is visible on the disk all the time, we can expect that the filament is flying in our direction perpendicularly to some extent, so there would not be such a large difference between radial velocity and line-of-sight velocity. We expect that the radial velocity can be larger than the line-of-sight velocity if we assume the projection effect, while it will be about $\sqrt{ } 2$ times smaller at most if it erupts at a $45^{\circ}$ tilt in the radial direction, which does not change our discussion. The solid line indicates the threshold that can roughly distinguish filament eruption with and without CMEs derived in ref. ${ }^{30}$. The threshold can be expressed as $\left(V_{r_{-} \max } / 100 \mathrm{~km} \mathrm{~s}^{-1}\right)(\mathrm{L} / 100 \mathrm{Mm})^{0.96}=0.8$, which is determined by using the Linear Support Vector Classification algorithm (see ref. ${ }^{30}$ for the detailed method).

\section{Methods}

TESS light-curve analysis. TESS observed EK Dra (TIC 159613900) in its sectors 14-16 (18 July 2019-6 October 2020) and 21-23 (21 January 2020-15 April 2020). The TESS light curve from the $2 \mathrm{~min}$ time-cadence photometry was processed by the Science Processing Operations Center pipeline, a descendant of the Kepler mission pipeline based at the NASA Ames Research Center ${ }^{12,31}$. Extended Data Fig. 1 shows the light curve of EK Dra from BJD 2458945 (JD 2458944.997, 5 April 2020 11:56 UT; Sector 23), and the stellar superflare detected by TESS, the Seimei telescope and the Nayuta telescope in Fig. 1 is indicated with the red arrow in this figure. The quasiperiodic brightness variation is thought to be caused by the rotation of EK Dra with the asymmetrically spotted hemisphere ${ }^{3,5}$. The rotation period is reported as about $2.8 \mathrm{~d}\left(\right.$ ref. $\left.^{9}\right)$. Although the superflare occurred near the local brightness maximum, some of the starspots are expected to be visible from the observer ${ }^{32-35}$. In Extended Data Fig. 1, other flares are also indicated using black arrows with more than two consecutive observational points whose flaring amplitude is more than three times the TESS photometric errors ${ }^{3,36}$. The white-ligh flare energy was calculated by assuming the $10,000 \mathrm{~K}$ blackbody spectra ${ }^{36,37}$ (Flare energy). The pixel-level data analysis is shown in TESS pixel-level data analysis. The estimated occurrence frequency of superflares $\left(>10^{33} \mathrm{erg}\right)$ in the TESS band was about once per $2 \mathrm{~d}$, which means that about 12 nights' monitoring observations are necessary on average to detect one superflare from the ground-based telescope under a clear-sky ratio of $50 \%$. This implies that our datasets are highly unique.

Spectroscopic data analysis. Here, we present the utilization of low-resolution spectroscopic data from KOOLS-IFU ${ }^{38}$ of the $3.8 \mathrm{~m}$ Seimei telescope ${ }^{13}$ at Okayama Observatory of Kyoto University and MALLS ${ }^{19,39}$ of the $2 \mathrm{~m}$ Nayuta telescope at Nishi-Harima Astronomical Observatory of the University of Hyogo. KOOLS-IFU is an optical spectrograph with a spectral resolution of $R(\lambda / \Delta \lambda) \sim 2,000$ covering a wavelength range from 5,800 to $8,000 \AA$; it is equipped with Ne gas emission lines for wavelength calibration and instrument characterization. The exposure time was set to be $30 \mathrm{~s}$ for this night. The sky spectrum was subtracted by using the sky fibres for each spectrum. The data reduction follows the prescription in ref. ${ }^{40}$. During this observation, the signal-to-noise ratio $(\mathrm{S} / \mathrm{N})$ for one frame is typically $172 \pm 6$. The observations using the Seimei telescope ended just after $133.7 \mathrm{~min}$ (Fig. 1b-d).

MALLS is an optical spectrograph with $R \sim 10,000$ at the H $\alpha$ line covering a wavelength range from 6,350 to 6,800 $\AA$; it is also equipped with Fe, Ne and Ar gas emission lines for wavelength calibration and instrument characterization. The sky spectrum was subtracted using a nearby region along the slit direction for each observation. The exposure time was set to be $3 \mathrm{~min}$ for this night. The MALLS data reduction follows the prescription in ref. ${ }^{19}$. The $\mathrm{S} / \mathrm{N}$ for one frame is typically $86 \pm 8$ during this observation. For the MALLS data, the wavelength corrections are also performed for each spectrum using the Earth's atmospheric absorption lines.

We corrected the wavelength for the proper motion velocity of $-20.7 \mathrm{~km} \mathrm{~s}^{-1}$ of EK Dra on the basis of Gaia Data Release 2 (ref. ${ }^{41}$ ). Continuum levels are defined by a linear fit between the wavelength ranges of the $\mathrm{H} \alpha$ line wing $(6,517.8-6,537.8$ and $6,587.8-6,607.8 \AA$ ). We take the continuum level as the wavelength range between $6,517.8-6,537.8$ and $6,587.8-6,607.8 \AA$ to measure the EW $\left(=\int\left(1-F_{\lambda} / F_{0}\right) \mathrm{d} \lambda\right.$, where $F_{0}$ is the continuum intensity on either side of the absorption feature, while $F_{\lambda}$ represents the intensity across the entire wavelength range of interest). The original spectra are shown in Stability of pre-flare spectra. Extended Data Fig. 2 shows the pre-flare-subtracted $\mathrm{H} \alpha$ spectra during and after the superflare on EK Dra with higher time cadence than Fig. 1e. The narrowband $\mathrm{H} \alpha \mathrm{EW}(\mathrm{H} \alpha-10 \AA-\mathrm{H} \alpha+10 \AA)$ is used for the measurements of the radiated energy and duration of the $\mathrm{H} \alpha$ flare because of the high $\mathrm{S} / \mathrm{N}$, and the broadband $\mathrm{H} \alpha \mathrm{EW}(\mathrm{H} \alpha-20 \AA-\mathrm{H} \alpha+10 \AA)$ is used for the measurements of the amount of absorption (that is, mass and kinetic energy)

Solar data analysis. In the main text, we showed the data of a C5.1-class solar flare (that is, the peak GOES soft X-ray flux $F_{\mathrm{GOES}}$ is $5.1 \times 10^{-6} \mathrm{~W} \mathrm{~m}^{-2}$; hereafter 'Event 1 ', see Table 1) and associated filament eruption around 07:56 UT, 7 July 2016, observed using the SDDI ${ }^{15}$ installed on the SMART at Hida Observatory (further explanations continue to Solar data analysis (continued from Methods)). The SDDI conducted a monitoring observation of the Sun on 7 July 2016. It takes full-disk solar images at 41 wavelength points every $0.5 \AA$ from the H $\alpha$ line centre $-8.0 \AA$ $\left(-366 \mathrm{~km} \mathrm{~s}^{-1}\right)$ to the $\mathrm{H} \alpha$ line centre $+8.0 \AA\left(366 \mathrm{~km} \mathrm{~s}^{-1}\right)$, while it takes images every $0.25 \AA$ from the $\mathrm{H} \alpha$ line centre $-2.0 \AA\left(-91 \mathrm{~km} \mathrm{~s}^{-1}\right)$ to the $\mathrm{H} \alpha$ line centre $+2.0 \AA$ $\left(91 \mathrm{~km} \mathrm{~s}^{-1}\right)$. Each set of images is obtained with a time cadence of $20 \mathrm{~s}$ and a pixel size of about 1.2 arcsec. The SDDI started the daily monitoring observations in 2016, and the C5.1-class solar flare is one of the largest solar flares with a filament eruption among the events observed by SDDI with good weather conditions in these five years. The solar filament eruption was also reported in refs. ${ }^{15,42}$. Another jet-like filament eruption (known as a solar surge ${ }^{1}$ ) associated with a C8.0-class solar flare is also shown in Another case of solar flares on 2 April 2017 (hereafter we call this surge 'Event 2', see Table 1).

This paper used $70 \mathrm{~min}$ time series of the SDDI images taken from 07:30 UT on 7 July 2016 (Supplementary Video 1). As in Extended Data Fig. 4, the C5.1-class flare occurred around an active region, named 'NOAA 12561', on the solar disk, and was accompanied by a typical filament eruption ${ }^{15,42}$. The spectra from the event are integrated over a spatial region that is large enough to cover the visible phenomena (the magenta region in Extended Data Fig. 4a,b). The spectra are reconstructed by using the template solar $\mathrm{H} \alpha$ spectrum convolved with the SDDI instrumental profile.

Here, we define $L(\lambda, t, A)$ as the luminosity at a wavelength of $\lambda$ and time of $t$ that is integrated for the region $A$ (that is, $L(\lambda, t, A)=\int_{A} I(t) \mathrm{d} A ; I(t)$ is intensity). We now define $A_{\text {local }}$ as the integration region (magenta region in Extended Data Fig. 4a,b), and $A_{\text {full-disk }}$ as the solar full disk. We first obtain the local (partial-image) pre-flare-subtracted spectra $\Delta S_{\text {local }}$, which are normalized by the local (partial-image) total continuum level $\left(L\left(6,570.8 \AA, t, A_{\text {local }}\right)\right)$ :

$$
\Delta S_{\text {local }}=\frac{L\left(\lambda, t, A_{\text {local }}\right)-L\left(\lambda, t_{0}, A_{\text {local }}\right)}{L\left(6,570.8 \AA, t, A_{\text {local }}\right)},
$$

where $t_{0}$ is a given time of the pre-flare period. Then, the (virtual) full-disk pre-flare-subtracted spectra $\Delta S_{\text {full-disk }}$ are obtained by multiplying by the ratio of the partial-image continuum to full-disk continuum (total continuum ratio):

$$
\Delta S_{\text {full-disk }}=\Delta S_{\text {local }} \frac{L\left(6,570.8 \AA, t_{0}, A_{\text {local }}\right)}{L\left(6,570.8 \AA, t_{0}, A_{\text {full-disk }}\right)},
$$

and we obtain a virtual pre-flare-subtracted spectrum of this phenomenon as if we observed the Sun as a star. The EW of the H $\alpha$ is also calculated using the $\Delta S_{\text {full-disk, }}$, and we obtained the virtual Sun-as-a-star $\Delta \mathrm{H} \alpha \mathrm{EW}$ (that is, differential $\mathrm{H} \alpha$ flux normalized by the full-disk continuum level).

Stellar velocity, mass and kinetic energy data. For the stellar filament eruption, the velocity is derived by fitting the absorption spectra obtained using the Seime telescope with the normal distribution $N\left(\lambda, \mu, \sigma^{2}\right)$, where $\mu$ is the mean wavelength and $\sigma^{2}$ is the variance. In Extended Data Fig. 3a, we plotted the temporal evolution of the velocity $((\mu-\lambda) / \lambda c$, where $\lambda$ is $6,562.8 \AA$ and $c$ is light speed) for the fitted absorption feature with the width of $\sigma$. We only plotted the data whose absorption 
Table 1 | Properties of solar filament eruptions/surges reported in previous studies and this study. The data are plotted in Fig. 3 . The calculation of flare energy, mass and kinetic energy is introduced in Velocity, mass, and kinetic energy: solar data

\begin{tabular}{|c|c|c|c|c|c|c|}
\hline Date yyyy/mm/dd & GOES & Flare energy $\left(10^{29} \mathrm{erg}\right)$ & Mass (g) & Kinetic energy (erg) & Reference & Event \\
\hline $1980 / 10 / 30$ & C4.8 & 4.5 & $10^{14-15}$ & $3.14 \times 10^{28}$ & 79 & surge \\
\hline $2001 / 08 / 30$ & C5.8 & 5.5 & - & $1.4 \times 10^{27}$ & 80 & surge \\
\hline $1993 / 05 / 14$ & M4.4 & 44 & $10^{15}$ & $1.5 \times 10^{29}$ & 81 & filament eruption \\
\hline 2012/02/11 & $\mathrm{C} 2.7$ & 2.7 & $4 \times 10^{11-13}$ & $2 \times 10^{28}$ & 82 & filament eruption \\
\hline 2016/07/07 & C5.1 & 5.1 & $1.3 \times 10^{15}$ & $7.7 \times 10^{28}$ & Event 1 & filament eruption \\
\hline $2017 / 04 / 02$ & C8.0 & 8.0 & $5.1 \times 10^{14}$ & $8.5 \times 10^{27}$ & Event $2^{\mathrm{a}}$ & surge \\
\hline
\end{tabular}

a The analysis of the solar surge (Event 2) is described in Another case of solar flares on 2 April 2017.

features are clear enough to fit the shape with the threshold of the fitted absorption amplitude of $>0.01$ and fitted velocity dispersion of $<500 \mathrm{~km} \mathrm{~s}^{-1}$ and $>100 \mathrm{~km} \mathrm{~s}^{-1}$. The threshold was determined by trial and error, and we find that many missed detections of absorption features occur when we select threshold values other than this one. The amplitude value of 0.01 corresponds to the detection limit when considering the typical S/N $\sim 170$ of the Seimei telescope/KOOLS-IFU, and the lower limit of $100 \mathrm{~km} \mathrm{~s}^{-1}$ is determined to avoid detecting the sharp noisy signals. About $27 \%$ of data points were discarded due to this threshold from the initial points $(22 \mathrm{~min})$ to final points $(110 \mathrm{~min})$, especially for the latter decaying phase. Here, the maximum observed velocity and its errors are calculated as $510 \pm 120 \mathrm{~km} \mathrm{~s}^{-1}$ with a width of $220 \pm 90 \mathrm{~km} \mathrm{~s}^{-1}$ from the mean values of the $\mu$ and $\sigma$ of the first five points ( $t=22-26 \mathrm{~min}$ in Fig. 1 ), respectively. The mean value of the velocity when the absorption becomes strong $(t=25-50$ min in Fig. 1$)$ is estimated as $258 \mathrm{~km} \mathrm{~s}^{-1}$

The plasma mass is simply calculated from the total $\mathrm{H} \alpha \mathrm{EW}$. We used the simple Becker cloud model ${ }^{43}$ with optical depth at the line centre of the ejected plasma $\tau_{0}$ of 5 (which is slightly more optically thick than solar filament eruptions; compare ref. ${ }^{44}$ ), two-dimensional aspect ratio of 1 (that is, cubic), local plasma dispersion velocity $W$ of $20 \mathrm{~km} \mathrm{~s}^{-1}$ and source function $S$ of 0.1 on the basis of the solar observations ${ }^{45}$. The observed half-width of $220 \mathrm{~km} \mathrm{~s}^{-1}$ of the stellar blueshifted component is larger by one order of magnitude than the solar value, but here we use the solar value as a template. The dispersion velocity of $220 \mathrm{~km} \mathrm{~s}^{-1}$ is considered to be the upper limit of the local velocity dispersion because the ejected mass would have a complex two-dimensional velocity distribution, which can cause larger $W$ in the integrated spectra. First, the modelled EW of enhanced absorption is calculated by using the Becker cloud model, when the plasma velocity $v_{\text {shift }}$ is $-258 \mathrm{~km} \mathrm{~s}^{-1}$, as

$$
\begin{gathered}
\text { model EW }=\int_{\lambda} \frac{I_{\lambda}-I_{0 \lambda}}{I_{0, \text { Cont. }}} \mathrm{d} \lambda=\int_{\lambda} \frac{S-I_{0 \lambda}}{I_{0, \text { Cont. }}}\left(1-\mathrm{e}^{-\tau_{\lambda}}\right) \mathrm{d} \lambda \\
\tau_{\lambda}=\tau_{0} \exp \left[-\frac{1}{2}\left(\frac{\lambda / \lambda_{0}-\left(1+v_{\text {shift }} / c\right)}{W / c}\right)^{2}\right],
\end{gathered}
$$

where $I_{0 \lambda}$ is background intensity and $I_{0, \text { Cont. }}$ is continuum intensity. This is the EW value for an extreme case when the full disk of the star is completely covered with absorbing, cool ejected plasma. By comparing the modelled EW (equation (3)) with the lowest observed stellar EW value of $-0.16 \AA$ (integrated for $\mathrm{H} \alpha-20 \AA-\mathrm{H} \alpha+10 \AA$; Supplementary Fig. 4c), the cool-plasma filling factor is calculated to be $5.9 \%$ of the stellar disk (that is, modelled EW/observed EW; area $=1.6 \times 10^{21} \mathrm{~cm}^{2}$ ). Using the length scale of the ejected plasma, $3.9 \times 10^{10} \mathrm{~cm}$ $\left(=\right.$ area $\left.^{0.5}\right)$, the hydrogen column density is derived as $4.0 \times 10^{20} \mathrm{~cm}^{-2}$ from the assumed optical depth based on the plasma model ${ }^{46}$. In the model of ref. ${ }^{46}$, hydrogen/electron density is calculated by assuming an ionization equilibrium for a population of hydrogen atoms due to a balance between recombination and radiative photoionization through the Balmer/Lyman continuum. It should be noted that the ionization equilibrium of filaments on active stars may be somewhat different from the solar observations due to their high UV radiations, which may affect the evaluation of the mass of the ejecta. By multiplying the hydrogen column density by the filament area, we then obtained the plasma mass of $1.1 \times 10^{18} \mathrm{~g}$. If the two-dimensional aspect ratio becomes 0.1 , similar to a jet-like feature ( $x$ width: $y$ width: $z$ depth $=1: 0.1: 0.1$ ), then the estimated mass becomes larger by a factor of 1.78 . If optical depth ranges from 0.8 to 10 (ref. ${ }^{44}$ ), the source function takes values of 0.02 or 0.5 and the dispersion velocity is 10 or $220 \mathrm{~km} \mathrm{~s}^{-1}$ (ref. ${ }^{45}$ ), then the estimated masses change by a factor between 0.15 and 4.9. In Fig. 3a, we used the mass of $1.1_{-0.9}^{+4.2} \times 10^{18} \mathrm{~g}$ for an optical depth of 5 , and the uncertainties of the model (0.15-4.9) are used as the error bars since the model-based errors are expected to be much larger than the observational errors. It should be noted that this mass estimate could be either a significant overestimate of the mass of an affiliated CME due to most of the filament falling back to the star, or a significant underestimate due to most of the CME actually being hot coronal material rather than cool filament. The plasma kinetic energy is then calculated as $3.5_{-3.0}^{+14.0} \times 10^{32} \mathrm{erg}$ by using the velocity of $258 \mathrm{~km} \mathrm{~s}^{-1}$. The observed maximum velocity was $510 \mathrm{~km} \mathrm{~s}^{-1}$ in the early phase, so the kinetic energy can be larger by a factor of 4 although the absorption component was weak at that time.

Related works on candidate stellar filament eruptions or CMEs on other types of star. Here, we discuss potential stellar filament eruptions/CMEs reported in the previous studies (see refs. ${ }^{6,24,47}$ for reviews). In other stars, such as M-type stars $^{18-21,21,48-56}$, K-type stars ${ }^{57}$, T Tauri stars ${ }^{58,59}$, close binaries ${ }^{60,61}$ and giant stars ${ }^{23}$ some observational candidates for stellar filament eruptions/CMEs have been reported, although confirmations of filament eruptions/CMEs in analogy with solar observations are still rare. We also note that some other studies have tried to detect a signature of stellar filament eruptions/CMEs in various ways but have not succeeded in robust detection ${ }^{62-69}$.

A CME signature was reported from a blueshifted emission component of the cool X-ray O vir line (4MK) in the late phase of a stellar flare on the evolved giant star HR $9024^{23}$. Although the time evolution of the blueshifted velocity is not obtained there, they detected the blueshifted emission component with a velocity of $90 \mathrm{~km} \mathrm{~s}^{-1}$ (escape velocity $220 \mathrm{~km} \mathrm{~s}^{-1}$ ) and interpreted it as a CME. The blueshifted plasma components at a few MK are also emitted from the upward flow in the confined flare loops (called 'chromospheric evaporation') in the case of solar flares, but they exclude the possibility considering that the other hotter lines do not show the blueshifted component in the post-flare phase. Although the spectral type of HD 9024 (evolved giant star) is very different from that of EK Dra and the velocity $\left(90 \mathrm{~km} \mathrm{~s}^{-1}\right)$ is smaller than our observation $\left(510 \mathrm{~km} \mathrm{~s}^{-1}\right)$, the two observations share the trend that the mass ejection signature is dominant in the post-flare phase.

Blueshifted emission components of chromospheric lines have been reported in association with Balmer-line flares mostly on active $\mathrm{M} / \mathrm{K}$ dwarfs ${ }^{18-21,48-57}$ (see refs. ${ }^{24,44}$ for a summary). Time-varying blueshifted hydrogen emission components have also been reported with high time cadence on $\mathrm{M}$ dwarfs (for example, refs. ${ }^{19,21}$ ). A similar case is reported for a UV flare on an $\mathrm{Mdwarf} \mathrm{f}^{20,70}$. These may be evidence of stellar prominence eruptions/CMEs. It seems quite possible that the blueshifted emission lines on M dwarfs are closely analogous to the $\mathrm{H} \alpha$ absorption signatures studied in this Letter. The fundamental difference between G-dwarf and M-dwarf blueshift signatures is that for hotter G dwarfs $\mathrm{H} \alpha$ in an erupting filament will only be detectable in absorption, whereas for the cooler $M$ dwarfs even the quiescent $\mathrm{H} \alpha$ line is in emission, so an erupting filament might be observed in emission as well (compare ref. ${ }^{44}$ ). Blue-wing enhancements of M-dwarf flares are characterized by high velocity of several hundred kilometres per second (sometimes more than this) $)^{18,53,55}$, which cannot be explained by chromospheric evaporation flow associated with the chromospheric-line blueshift phenomenon observed in solar flares ${ }^{16,44,71-74}$. The high velocities of M-dwarf flares are similar to that detected on EK Dra in this study $\left(\sim 510 \mathrm{~km} \mathrm{~s}^{-1}\right)$. In addition, not all but some of the blueshift events on $\mathrm{M}$ dwarfs appear after the impulsive phase $\mathrm{e}^{20,21}$, which shares properties with filament eruption events on EK Dra and the Sun in this study. Therefore, at present the blueshifted emission lines in M-type stars are most probably prominence eruptions.

Other signatures of kinematic characteristics of the ejected plasma are also inferred from continuous X-ray absorption during stellar flares, which can be caused by neutral material above the flaring region, such as filament eruptions ${ }^{24,59,60,75-83}$. However, on the Sun, X-ray absorption by prominences is uncommon $^{44,84}$, and instrumental calibration effects at low energy have been pointed out ${ }^{47}$.

In some cases of binary stars, eclipses of the white dwarf component have been interpreted as obscuration by stellar mass ejected from the late-type companion $\operatorname{star}^{61,85}$. Other than this, pre-flare dips have been reported in stellar flares, suggesting potential prominence eruptions/CMEs ${ }^{86,87}$. Radio observations have recently investigated the type II radio bursts associated with shocks in front of CMEs as possible indirect evidence of CMEs, but no significant signature has been obtained so far ${ }^{47,62-65,67-69}$. Recently, a stellar type IV burst event from the M-type star Proxima Centauri was reported and may be evidence for a stellar $\mathrm{CME}^{56}$. 


\section{Data availability}

Source data are provided with this paper. In addition, all raw spectroscopic data are available either in the associated observatory archive (https://smoka.nao.ac.jp/ index.jsp for KOOLS-IFU data in Fig. 1 (available after January 2022); https://www. hida.kyoto-u.ac.jp/SMART/T1.html for some of the SDDI data in Fig. 2) or upon request from the corresponding author (for MALLS data in Fig. 1 and full raw data of SDDI). The TESS light curve is available at the MAST archive (https://mast.stsci. edu/portal/Mashup/Clients/Mast/Portal.html).

Received: 10 June 2021; Accepted: 1 October 2021;

Published online: 9 December 2021

\section{References}

1. Shibata, K. \& Magara, T. Solar flares: magnetohydrodynamic processes. Living Rev. Sol. Phys. 8, 6 (2011).

2. Gopalswamy, N. et al. Prominence eruptions and coronal mass ejection: a statistical study using microwave observations. Astrophys. J. 586, 562-578 (2003).

3. Maehara, H. et al. Superflares on solar-type stars. Nature $\mathbf{4 8 5}$, 478-481 (2012).

4. Shibata, K. et al. Can superflares occur on our Sun? Publ. Astron. Soc. Jpn. 65 49 (2013).

5. Notsu, Y. et al. Do Kepler superflare stars really include slowly rotating Sun-like stars?-Results using APO $3.5 \mathrm{~m}$ telescope spectroscopic observations and Gaia-DR2 data. Astrophys. J. 876, 58 (2019).

6. Airapetian, V. S. et al. Impact of space weather on climate and habitability of terrestrial-type exoplanets. Int. J. Astrobiol. 19, 136-194 (2020).

7. Osten, R. A. \& Wolk, S. J. Connecting flares and transient mass-loss events in magnetically active stars. Astrophys. J. 809, 79 (2015).

8. Miyake, F. et al. A signature of cosmic-ray increase in AD 774-775 from tree rings in Japan. Nature 486, 240-242 (2012).

9. Waite, I. A. et al. Magnetic fields on young, moderately rotating sun-like stars-II. EK Draconis (HD 129333). Mon. Not. R. Astron. Soc. 465, 2076-2091 (2017)

10. Ayres, T. R. The flare-ona of EK Draconis. Astrophys. J. 150, 7 (2015).

11. Audard, M., Güdel, M. \& Guinan, E. F. Implications from extreme-ultraviolet observations for coronal heating of active stars. Astrophys. J. 513, L53 (1999).

12. Ricker, G. R. et al. Transiting Exoplanet Survey Satellite (TESS). J. Astron. Telesc. Instrum. Syst. 1, 014003 (2014).

13. Kurita, M. et al. The Seimei telescope project and technical developments. Publ. Astron. Soc. Jpn. 72, 48 (2020).

14. Schmieder, B. et al. Evidence for gentle chromospheric evaporation during the gradual phase of large solar flares. Astrophys. J. 317, 956 (1987).

15. Ichimoto, K. et al. A new solar imaging system for observing high-speed eruptions: Solar Dynamics Doppler Imager (SDDI). Sol. Phys. 292, 63 (2017).

16. Švestka, Z., Kopecký, M. \& Blaha, M. Qualitative discussion of 244 flare spectra. II. Line asymmetry and helium lines. Bull. Astron. Inst. Czechoslov. 13, 37-42 (1962)

17. Ichimoto, K. \& Kurokawa, H. H $\alpha$ red asymmetry of solar flares. Sol. Phys. 93, 105-121 (1984).

18. Houdebine, E. R., Foing, B. H. \& Rodono, M. Dynamics of flares on late-type dMe stars. I. Flare mass ejections and stellar evolution. Astron. Astrophys. 238, 249 (1990).

19. Honda, S. et al. Time-resolved spectroscopic observations of an M-dwarf flare star EV Lacertae during a flare. Publ. Astron. Soc. Jpn 70, 62 (2018).

20. Leitzinger, M. et al. Search for indications of stellar mass ejections using FUV spectra. Astron. Astrophys. 536, A62 (2011)

21 . Vida, K. et al. Investigating magnetic activity in very stable stellar magnetic fields. Long-term photometric and spectroscopic study of the fully convective M4 dwarf V374 Pegasi. Astron. Astrophys. 590, A11 (2016).

22. Veronig, A. M. et al. Indications of stellar coronal mass ejections through coronal dimmings. Nat. Astron. 5, 697-706 (2021).

23. Argiroffi, C. et al. A stellar flare-coronal mass ejection event revealed by X-ray plasma motions. Nat. Astron. 3, 742-748 (2019).

24. Moschou, S.-P. et al. The stellar CME-flare relation: what do historic observations reveal? Astrophys. J. 877, 105 (2019).

25. Wood, B. E., Howard, R. A. \& Linton, M. G. Imaging prominence eruptions out to $1 \mathrm{AU}$. Astrophys. J. 816, 67 (2016).

26. Aarnio, A. N., Matt, S. P. \& Stassun, K. G. Mass loss in pre-main-sequence stars via coronal mass ejections and implications for angular momentum loss. Astrophys. J. 760, 9 (2012).

27. Drake, J. J. et al. Implications of mass and energy loss due to coronal mass ejections on magnetically active stars. Astrophys. J. 764, 170 (2013).

28. Takahashi, T., Mizuno, Y. \& Shibata, K. Scaling relations in coronal mass ejections and energetic proton events associated with solar superflares. Astrophys. J. 833, L8 (2016).
29. Alvarado-Gómez, J. D. et al. Suppression of coronal mass ejections in active stars by an overlying large-scale magnetic field: a numerical study. Astrophys. J. 862, 93 (2018).

30. Seki, D. et al. Relationship between three-dimensional velocity of filament eruptions and CME association. Earth Planets Space 73, 58 (2021).

31. Fausnaugh, M. M. et al. TESS Data Release Notes: Sector 23, DR32 (NASA, 2020); https://archive.stsci.edu/missions/tess/doc/tess_drn/tess_sector_23_ drn32_v03.pdf

32. Roettenbacher, R. M. \& Vida, K. The connection between starspots and flares on main-sequence Kepler stars. Astrophys. J. 868, 3 (2018).

33. Doyle, L. et al. Investigating the rotational phase of stellar flares on M dwarfs using K2 short cadence data. Mon. Not. R. Astron. Soc. 480, 2153-2164 (2018).

34. Doyle, L., Ramsay, G. \& Doyle, J. G. Superflares and variability in solar-type stars with TESS in the southern hemisphere. Mon. Not. R. Astron. Soc. 494, 3596-3610 (2020).

35. Namekata, K. et al. Temporal evolution of spatially resolved individual star spots on a planet-hosting solar-type star: Kepler-17. Astrophys. J. 891, 103 (2020).

36. Shibayama, T. et al. Superflares on solar-type stars observed with Kepler. I. Statistical properties of superflares. Astrophys. J. Suppl. Ser. 209, 5 (2013).

37. Namekata, K. et al. Statistical studies of solar white-light flares and comparisons with superflares on solar-type stars. Astrophys. J. 851, 91 (2017).

38. Matsubayashi, K. et al. Kools-IFU: Kyoto Okayama Optical Low-dispersion Spectrograph with optical-fiber integral field unit. Publ. Astron. Soc. Jpn 71, 102 (2019).

39. Ozaki, S. \& Tokimasa, N. The optical spectrograph for the NAYUTA telescope I. Design. Annu. Rep. Nishi-Harima Astron. Obs. 15, 15-29 (2005).

40. Namekata, K. et al. Optical and X-ray observations of stellar flares on an active M dwarf AD Leonis with the Seimei Telescope, SCAT, NICER, and OISTER. Publ. Astron. Soc. Jpn 72, 68 (2020).

41. Gaia Collaboration et al. Gaia Data Release 2. Summary of the contents and survey properties. Astron. Astrophys. 616, A1 (2018)

42. Seki, D. et al. SMART/SDDI filament disappearance catalogue. Sun Geosph. 14, 95-103 (2019).

43. Mein, P. \& Mein, N. Differential cloud models for solar velocity field measurements. Astron. Astrophys. 203, 162-169 (1988)

44. Odert, P. et al. Stellar coronal mass ejections-II. Constraints from spectroscopic observations. Mon. Not. R. Astron. Soc. 494, 3766-3783 (2020).

45. Sakaue, T. et al. Observational study on the fine structure and dynamics of a solar jet. II. Energy release process revealed by spectral analysis. Publ. Astron. Soc. Jpn. 70, 99 (2018).

46. Tsiropoula, G. \& Schmieder, B. Determination of physical parameters in dark mottles. Astron. Astrophys. 324, 1183-1189 (1997).

47. Osten, R. A. \& Wolk, S. J. A framework for finding and interpreting stellar CMEs. Proc. IAU Symp . 328, 243-251 (2017).

48. Gunn, A. G. et al. High-velocity evaporation during a flare on AT Microscopii. Astron. Astrophys. 285, 489-496 (1994).

49. Fuhrmeister, B. \& Schmitt, J. H. M. M. Detection and high-resolution spectroscopy of a huge flare on the old M 9 dwarf DENIS 104814.7-395606.1. Astron. Astrophys. 420, 1079-1085 (2004).

50. Fuhrmeister, B. et al. Multiwavelength observations of a giant flare on CN Leonis. I. The chromosphere as seen in the optical spectra. Astron. Astrophys. 487, 293-306 (2008).

51. Leitzinger, M. et al. A search for flares and mass ejections on young late-type stars in the open cluster Blanco-1. Mon. Not. R. Astron. Soc. 443, 898-910 (2014).

52. Korhonen, H. et al. Hunting for stellar coronal mass ejections. Proc. IAU Symp. 328, 198-203 (2017).

53. Vida, K et al. The quest for stellar coronal mass ejections in late-type stars. I. Investigating Balmer-line asymmetries of single stars in virtual observatory data. Astron. Astrophys. 623, A49 (2019).

54. Muheki, P. et al. Properties of flares and CMEs on EV Lac: possible erupting filament. Mon. Not. R. Astron. Soc. 499, 5047-5058 (2020).

55. Maehara, H. et al. Time-resolved spectroscopy and photometry of M dwarf flare star YZ Canis Minoris with OISTER and TESS: blue asymmetry in the $\mathrm{H} \alpha$ line during the non-white light flare. Publ. Astron. Soc. Jpn 73, 44-65 (2021).

56. Zic, A. et al. A flare-type IV burst event from Proxima Centauri and implications for space weather. Astrophys. J. 905, 23 (2020).

57. Flores Soriano, M. \& Strassmeier, K. G. Short-term evolution and coexistence of spots, plages and flare activity on LQ Hydrae. Astron. Astrophys. 597, A101 (2017)

58. Guenther, E. W. \& Emerson, J. P. Spectrophotometry of flares and short time scale variations in weak line, and classical T Tauri stars in Chamaeleon. Astron. Astrophys. 321, 803-810 (1997).

59. Tsuboi, Y. et al. ASCA detection of a superhot 100 million K X-ray flare on the weak-lined T Tauri star V773 Tauri. Astrophys. J. 503, 894 (1998). 
60. Favata, F. \& Schmitt, J. H. M. M. Spectroscopic analysis of a super-hot giant flare observed on Algol by BeppoSAX on 30 August 1997. Astron. Astrophys. 350, 900-916 (1999).

61. Bond, H. E. et al. Detection of Coronal Mass Ejections in V471 Tauri with the Hubble Space Telescope. Astrophys. J. 560, 919-927 (2001).

62. Leitzinger, M. et al. Stellar activity at FUV/radio wavelengths. Cent. Eur. Astrophys. Bull. 34, 123 (2010).

63. Boiko, A. I. et al. Search of the radio emission from flare stars at decameter wavelengths. Adv. Astron. Space Phys. 2, 121 (2012).

64. Villadsen, J., Hallinan, G. \& Bourke, S. Radio spectroscopy of stellar flares: magnetic reconnection \& CME shocks in stellar coronae. Proc. IAU Symp. 320, 191-195 (2016).

65. Crosley, M. K. et al. The search for signatures of transient mass loss in active stars. Astrophys. J. 830, 24 (2016).

66. Leitzinger, M. et al. A census of coronal mass ejections on solar-like stars. Mon. Not. R. Astron. Soc. 493, 4570-4589 (2020).

67. Crosley, M. K. \& Osten, R. A. Constraining stellar coronal mass ejections through multi-wavelength analysis of the active $\mathrm{M}$ dwarf EQ Peg. Astrophys. J. 862, 113 (2018).

68. Crosley, M. K. \& Osten, R. A. Low-frequency radio transients on the active M-dwarf EQ Peg and the search for coronal mass ejections. Astrophys. J. 856, 39 (2018).

69. Villadsen, J. \& Hallinan, G. Ultra-wideband detection of 22 coherent radio bursts on M dwarfs. Astrophys. J. 871, 214 (2019).

70. Ambruster, C. W. et al. An episode of mass expulsion from the M-dwarf flare star EV Lacertae? In ESA Proc. International Symposium on New Insights in Astrophysics. Eight Years of UV Astronomy with IUE (eds Rolfe, E. J. \& Wilson, R.) Vol. 263, 137-140 (ESA Special Publication, 1986).

71. Allred, J. C. et al. Radiative hydrodynamic models of the optical and ultraviolet emission from solar flares. Astrophys. J. 630, 573-586 (2005)

72. Tei, A. et al. Blue-wing enhancement of the chromospheric $\mathrm{Mg}$ in $\mathrm{h}$ and $\mathrm{k}$ lines in a solar flare. Publ. Astron. Soc. Jpn 70, 100 (2018).

73. Kuridze, D. et al. H $\alpha$ line profile asymmetries and the chromospheric flare velocity field. Astrophys. J. 813, 125 (2015).

74. Heinzel, P. et al. On the occurrence of blue asymmetry in chromospheric flare spectra. Sol. Phys. 152, 393-408 (1994).

75. Jain, R. \& Sorathia, B. Flare-associated surge prominence on 1980 October 30. Astron. Astrophys. 8, 295-303 (1987).

76. Liu, Y. \& Kurokawa, H. On a surge: properties of an emerging flux region. Astrophys. J. 610, 1136-1147 (2004).

77. Ohyama, M. \& Shibata, K. X-ray plasma ejection associated with $\mathrm{H} \alpha$ filament eruption. Proc. Nobeyama Symp . 479, 367-370 (1998).

78. Christian, D. J. et al. H $\alpha$ and EUV observations of a partial CME. Astrophys. J. 804, 147 (2015).

79. Haisch, B. M. et al. Coordinated Einstein and IUE observations of a disparitions brusques type flare event and quiescent emission from Proxima Centauri. Astrophys. J. 267, 280-290 (1983).

80. Ottmann, R. \& Schmitt, J. H. M. M. ROSAT observation of a giant X-ray flare on Algol: evidence for abundance variations? Astron. Astrophys. 307, 813-823 (1996).

81. Wheatley, P. J. ROSAT observations of V471 Tauri, showing that stellar activity is determined by rotation, not age. Mon. Not. R. Astron. Soc. 297 1145-1150 (1998).

82. Franciosini, E. et al. BeppoSAX observation of a large long-duration X-ray flare from UX Arietis. Astron. Astrophys. 375, 196-204 (2001).

83. Pandey, J. C. \& Singh, K. P. A study of X-ray flares-II. RS CVn-type binaries. Mon. Not. R. Astron. Soc. 419, 1219-1237 (2012).

84. Schwartz, P. et al. Prominence visibility in Hinode/XRT images. Astrophys. J. 807, 97 (2015).

85. Parsons, S. G. et al. Eclipsing post-common envelope binaries from the Catalina surveys. Mon. Not. R. Astron. Soc. 429, 256-268 (2013).

86. Giampapa, M. S. et al. A preflare diminution in the quiescent flux of EQ Pegasi. Astrophys. J. Lett. 252, L39 (1982).

87. Doyle, J. G. \& Collier Cameron, A. Mass ejections from the rapidly rotating M dwarf star GL 890. Mon. Not. R. Astron. Soc. 244, 291-293 (1990).

\section{Acknowledgements}

Some of the data presented here were obtained at the Okayama Observatory of Kyoto University, which is operated as a scientific partnership with the National Astronomical Observatory of Japan. We are grateful to the staff of Okayama Observatory, M. Kurita, M. Kino, F. Iwamuro, K. Ohta, H. Izumiura, K. Matsubayashi, D. Kuroda and T. Nagata, for the telescope and instrument development. Some of the data presented here were obtained at the Nishi-Harima Astronomical Observatory of the University of Hyogo through the framework of the Optical and Infrared Synergetic Telescopes for Education and Research (OISTER). This work and operations of OISTER were supported by the Optical and Near-Infrared Astronomy Inter-University Cooperation Program and Grants-in-Aid of the Ministry of Education. Some of the solar data presented here were obtained at the Hida Observatory of Kyoto University, which is partly supported by the Project for Solar-Terrestrial Environment Prediction (PSTEP). We are grateful to the staff of Hida Observatory for the instrument development and daily observations. We thank A. Asai and K. Otsuji for useful discussions on solar mass ejections. Funding for the TESS mission is provided by NASA's Science Mission directorate. We thank J.J. Drake and S. Yashiro for kindly providing the data of CMEs in their works. We thank A.F. Kowalski for carefully checking the English and content. We also thank Enago (www. enago.jp) for the English review. K.N. is supported by the JSPS Overseas Challenge Program for Young Researchers. Y.N. was supported by the JSPS Overseas Research Fellowship Program. We acknowledge the International Space Science Institute and the supported International Team 464: The Role of Solar and Stellar Energetic Particles on (Exo)Planetary Habitability (ETERNAL). This research is supported by JSPS KAKENHI grant numbers 18J20048, 21 J00316 (K.N.), 17K05400, 20K04032, 20H05643 (H.M.), 21J00106 (Y.N.), 20K14521 (K. Isogai), 21H01131 (K. Shibata, H.M., S.H., K. Ichimoto and D.N.) and $15 \mathrm{H} 05814$ (K. Ichimoto).

\section{Author contributions}

K.N. led the campaign observations, conducted the data analyses and wrote the draft of the manuscript; H.M. and S.H. contributed to the data analyses; K.N., H.M., S.H., S.O., K. Isogai and D.N. contributed to the observations at the Seimei telescope; J.T., M. Takayama, T.O., T.S., N.K. and M. Tozuka contributed to the observations at the Nayuta telescope; K. Ichimoto and T.T.I. are responsible for instruments and observations at the SMART; H.M., K.L.M., F.O., M.N., R.A., M.O. and K. Shiraishi contributed to the ground-based follow-up photometric observations; K.N., H.M., Y.N., D.S., K. Ichimoto and K. Shibata contributed to the data interpretation.

\section{Competing interests}

The authors declare no competing interests.

\section{Additional information}

Extended data is available for this paper at https://doi.org/10.1038/s41550-021-01532-8. Supplementary information The online version contains supplementary material available at https://doi.org/10.1038/s41550-021-01532-8.

Correspondence and requests for materials should be addressed to Kosuke Namekata. Peer review information Nature Astronomy thanks the anonymous reviewers for their contribution to the peer review of this work.

Reprints and permissions information is available at www.nature.com/reprints.

Publisher's note Springer Nature remains neutral with regard to jurisdictional claims in published maps and institutional affiliations.

\section{(c) (i) Open Access This article is licensed under a Creative Commons}

Attribution 4.0 International License, which permits use, sharing, adaptation, distribution and reproduction in any medium or format, as long as you give appropriate credit to the original author(s) and the source, provide a link to the Creative Commons license, and indicate if changes were made. The images or other third party material in this article are included in the article's Creative Commons license, unless indicated otherwise in a credit line to the material. If material is not included in the article's Creative Commons license and your intended use is not permitted by statutory regulation or exceeds the permitted use, you will need to obtain permission directly from the copyright holder. To view a copy of this license, visit http://creativecommons. org/licenses/by/4.0/.

(C) The Author(s) 2021 


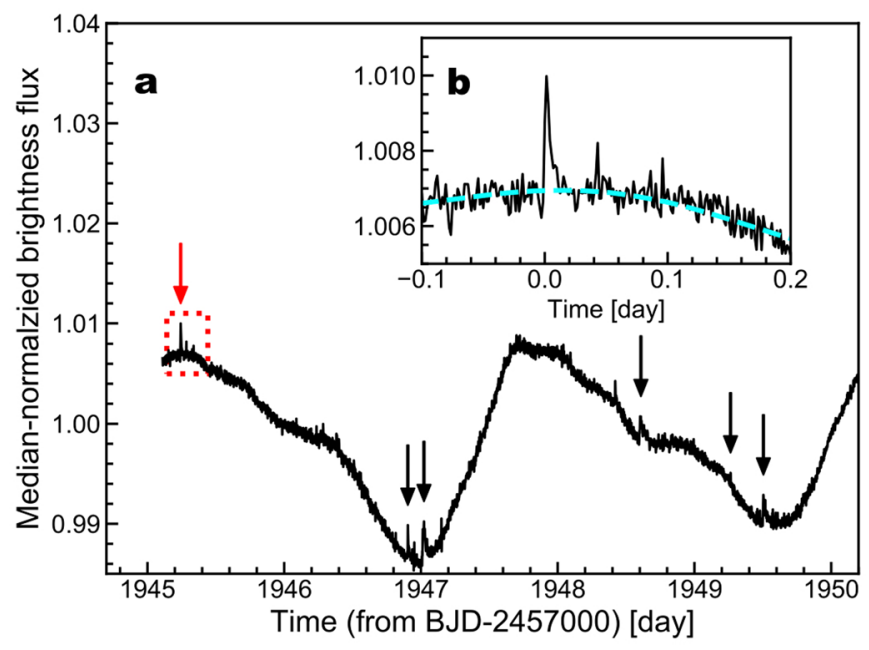

Extended Data Fig. 1 | Global TESS light curve for EK Dra. (a) Light curve of a superflare on EK Dra observed by TESS from BJD 2457000 . The gap before about BJD 2458945 days corresponds to a gap in the data downlink with Earth during the spacecraft's perigee. The arrows indicate stellar flares that occurred during this observational period, but the other flares in this figure were not observed by ground-based spectroscopic observations. The red arrow is the superflare shown in Fig. 1. (b) Enlarged light curve indicated with the red dotted box in panel (a). The cyan dashed line is the global trend of the light curve caused by the stellar rotation with large starspots. 

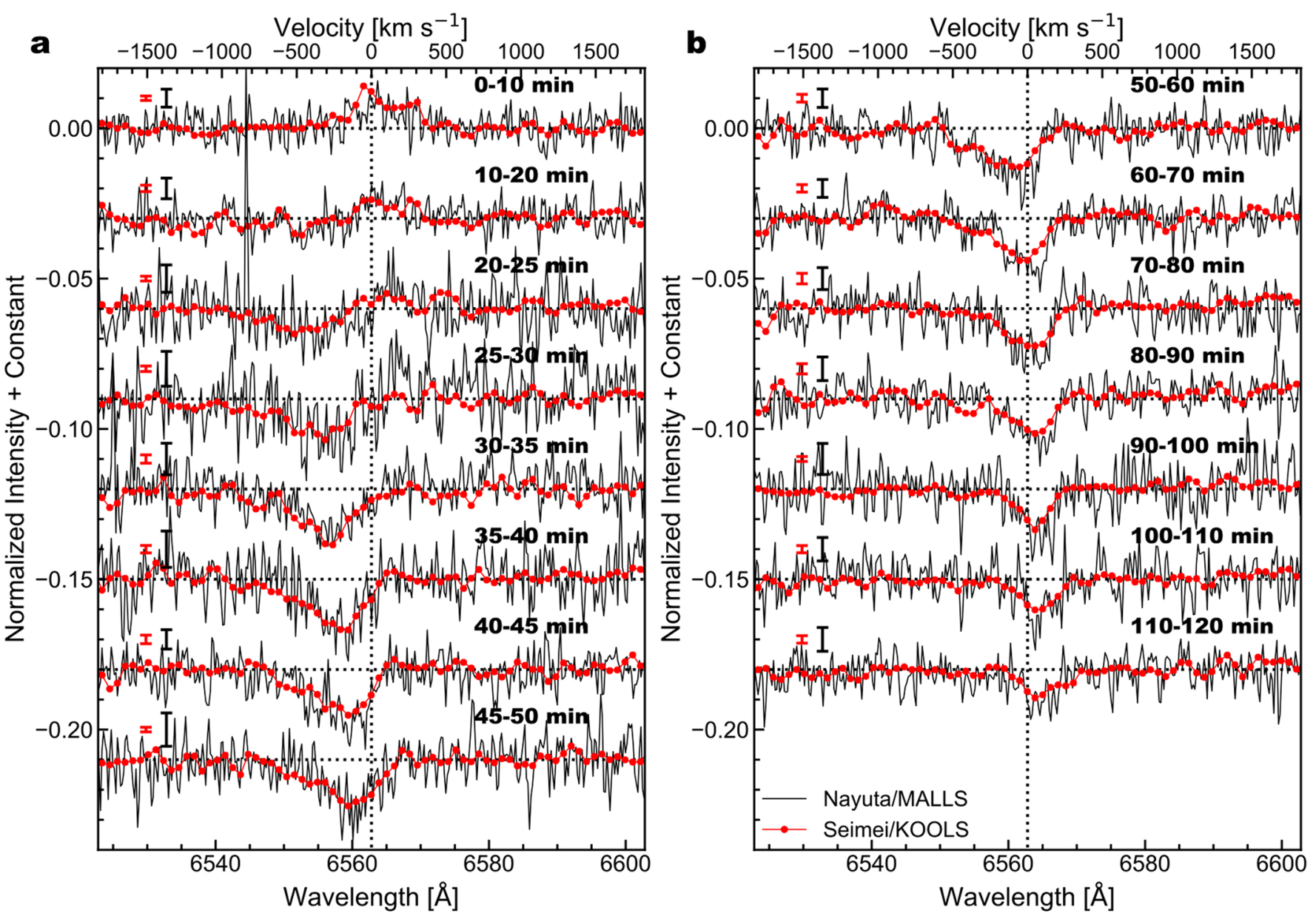

Extended Data Fig. 2 | Pre-flare-subtracted H $\alpha$ spectra during and after the superflare on EK Dra with higher time cadence than panel (e) in Fig. 1. (a, b) The red and black lines are the data observed by the Seimei telescope and the Nayuta telescope. The spectra are binned in time, and the integration periods correspond to the horizontal axes of panel (a-d) in Fig. 1. The intensities are normalized by the stellar continuum level. The vertical dotted line indicates the $\mathrm{H} \alpha$ line center, and the horizontal dotted lines indicate the zero levels for each spectrum. The $1-\alpha$ values for the line center are indicated with red (Seimei telescope) and black (Nayuta telescope) error bars for each time bin. The $1-\sigma$ values are basically calculated by the scattering in line wing (6522.8 - $6532.8 \AA$ and $6592.8-6602.8 \AA)$. 
a



b



Extended Data Fig. 3 | Temporal evolution of the velocity and deceleration for the $\mathbf{H} \boldsymbol{\alpha}$-line absorption features. (a) The blue points indicate the velocity of the $\mathrm{H} \alpha$-line absorption features seen after a superflare. The spectra observed by Seimei telescope were used considering the high $\mathrm{S} / \mathrm{N}$ and the absorption features are obtained by fitting them with a normal-distribution function. The error bars indicate the standard deviation of the fitted normal distribution. The green dashed line indicates the exponential function which fits the blue symbols, and the magenta dotted line indicates the velocity evolution of the free fall. In the free-fall model, we have numerically calculated the temporal change in velocity and height of material ejected vertically from the stellar surface at an initial velocity of $510 \mathrm{~km} / \mathrm{s}$ under the gravitational force $g(r(t))$ at that height $r(t)\left(\right.$ where $g(r(t))=G M / r(t)^{2}, t$ is time, $r(t)$ is the height of the filament at time $t, G$ is the constant of gravitation, $M_{\text {star }}$ is the mass of the star $0.95 M_{\odot}$ ). (b) The temporal evolution of deceleration rates is derived from the velocity changes for observation and free-fall model in panel (a). 

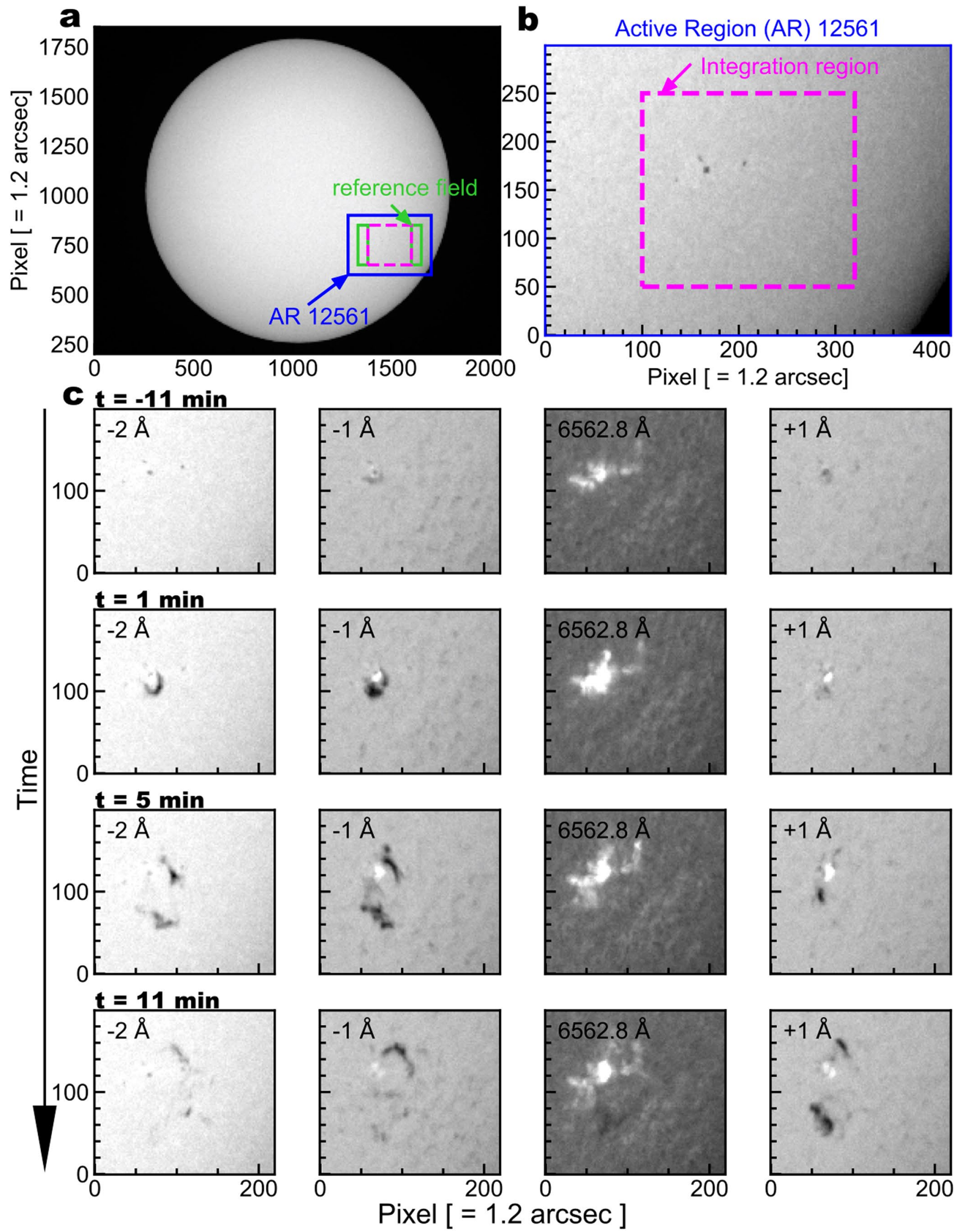

Extended Data Fig. 4 | See next page for caption. 
Extended Data Fig. 4 | A solar flare on 7 July, 2016, observed by SMART/SDDI at Hida observatory. (a) A full disk image of the Sun at the H $\alpha$ line wing

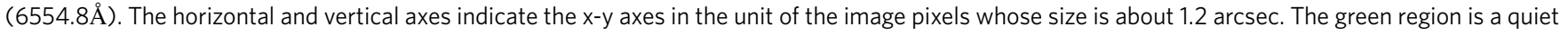
region used as a reference to make the $\mathrm{H} \alpha$ spectra. (b) the blue region is the enlarged panel of the active region 12561 . The magenta is the region where the solar flare and filament eruption happened. (c) The temporal evolution of solar images in the magenta region at a wavelength of $6560.8\left(-91 \mathrm{~km} \mathrm{~s}^{-1}\right)$, $6561.8\left(-46 \mathrm{~km} \mathrm{~s}^{-1}\right), 6562.8\left(0 \mathrm{~km} \mathrm{~s}^{-1}\right), 6563.8 \AA\left(+46 \mathrm{~km} \mathrm{~s}^{-1}\right)$. The emission and absorption features are indicated with white and black, respectively. The movie is shown in Supplementary Video 1. 